\title{
Resolving the Image of Gamma\#Ray Burst Afterglows with Gravitational Microlensing
}

\section{Citation}

Gaudi, B. Scott, and Abraham Loeb. 2001. "Resolving the Image of Gamma\#Ray Burst

Afterglows with Gravitational Microlensing." The Astrophysical Journal 558 (2): 643-56. https:// doi.org/10.1086/322289.

\section{Permanent link}

http://nrs.harvard.edu/urn-3:HUL.InstRepos:41393301

\section{Terms of Use}

This article was downloaded from Harvard University's DASH repository, and is made available under the terms and conditions applicable to Other Posted Material, as set forth at http:// nrs.harvard.edu/urn-3:HUL.InstRepos:dash.current.terms-of-use\#LAA

\section{Share Your Story}

The Harvard community has made this article openly available.

Please share how this access benefits you. Submit a story.

Accessibility 


\title{
Resolving the Image of Gamma-Ray Burst Afterglows with Gravitational Microlensing
}

\author{
B. Scott Gaudi ${ }^{1}$ \\ Institute for Advanced Study, Einstein Drive, Princeton, NJ 08540 \\ gaudi@sns.ias.edu \\ Abraham Loeb \\ Harvard-Smithsonian CfA, 60 Garden Street, Cambridge, MA 02138 \\ aloeb@cfa.harvard.edu
}

\begin{abstract}
Microlensing of a gamma-ray burst afterglow by an intervening star can be used to infer the radial structure of the afterglow image. Near the peak of the microlensing event, the outer edge of the image is more highly magnified than its central region, whereas the situation is reversed at later times due to the rapid radial expansion of the image on the sky. Thus, the microlensed afterglow light curve can be inverted to recover the self-similar radial intensity profile of the afterglow image. We calculate the expected errors in the recovered intensity profile as a function of the number of resolution elements, under the assumption that the afterglow and microlensing event parameters are known. For a point-mass lens and uniform source, we derive a simple scaling relation between these parameters and the resultant errors. We find that the afterglow need not be monitored for its entire duration; rather, observations from the peak magnification time $t_{\text {peak }}$ of the microlensing event until $\sim 7 t_{\text {peak }}$ are sufficient to resolve the majority of the afterglow image. Thus, microlensing events can be alerted by relatively infrequent observations of afterglows and then monitored intensively, without significant loss of information about the afterglow intensity profile. The relative intensity profile of $\sim 1 \%$ of all afterglows can be measured with 10 resolution elements to an accuracy of $\mathcal{O}(1 \%)$ in the optical and $\mathcal{O}(10 \%)$ in the infrared, using $4 \mathrm{~m}$-class telescopes. Weak microlensing events with large impact parameters are more common; we estimate that for $\sim 10 \%$ of afterglows the image profile may be inverted to a fractional accuracy $\lesssim 20 \%$ through frequent optical observations. We also calculate the effects of external shear due to the host galaxy or a binary companion, and contamination by background light from the host galaxy.
\end{abstract}


gamma rays:bursts - gravitational lensing

\section{Introduction}

Recent detections of afterglow emission in the X-ray, optical and radio has revolutionized gamma-ray burst (GRB) astronomy (for a recent review, see Kulkarni et al. 2000). The afterglow emission appears to be reasonably well-described by the fireball model (Waxman 1997a), in which a relativistically expanding shell of hot gas encounters an external quiescent medium. A blast wave is created, sweeping up the ambient material, and accelerating relativistic electrons which then emit synchrotron radiation (Paczynski \& Rhoads 1993; Meszaros \& Rees 1997). In this model, the spectral flux of the afterglow is a broken power-law function of frequency which evolves with time in a way that depends on the physical parameters of the fireball and its surrounding medium, such as the total energy output of the GRB source, the collimation angle of the outflow, the ambient gas density, the fraction of the post shock energy which is converted into magnetic fields and accelerated electrons, and the energy distribution of the accelerated electrons (Sari, Piran, \& Narayan 1999). So far, observations of the afterglow spectrum over a wide range of frequencies and times was the primary method used to place constraints on these parameters (e.g. Wijers \& Galama 1999; Panaitescu \& Kumar 2001; Freedman \& Waxman 1999). Crude constraints on the size of the emitting region at late times were derived based on considerations involving synchrotron self-absorption (Katz \& Piran 1997) and radio scintillations due to the intervening interstellar medium of the Milky-Way galaxy (Goodman 1997; Waxman, Kulkarni, \& Frail 1998).

The afterglow image is predicted to appear as a thin ring on the sky (Waxman 1997c; Sari 1998; Panaitescu \& Meszaros 1998) at frequencies near or above the peak of the synchrotron emission (Granot, Piran \& Sari 1999a), but more like a uniform disk around or below the synchrotron self-absorption frequency (Granot, Piran \& Sari 1999b). In between breaks in the power-law spectrum of an afterglow, the contrast and width of this ring evolve selfsimilarly, i.e. the brightness profile maintains its shape as a function of radius when the latter is normalized by the expanding circular boundary of the image (Granot, Piran \& Sari 1999a). The self-similar brightness profile of the image changes only across spectral breaks (Granot \& Loeb 2001).

The outer radius of the image expands radially at an apparent superluminal speed of $\sim \Gamma c$, where $\Gamma$ is the Lorentz factor of the emitting shock. The image occupies an angle of $1 / \Gamma$ relative to the center of the explosion due to relativistic beaming, and hence has a

\footnotetext{
${ }^{1}$ Hubble Fellow
} 
radius $R_{\mathrm{s}} \propto r_{\mathrm{sh}} / \Gamma$, where $r_{\mathrm{sh}}$ is the shock radius. Geometric time delay implies that the observed time, $t \propto r_{\mathrm{sh}} / \Gamma^{2}$, and so the radius scales as $R_{\mathrm{s}} \propto \Gamma t$. For an ambient density profile $\rho \propto r^{-k}$, one gets $\Gamma \propto r_{\text {sh }}^{-(3-k) / 2}$. Therefore, $R_{\mathrm{s}} \propto t^{\delta}$ with $\delta \equiv(5-k) /[2(4-k)]$. For a uniform ambient medium with $k=0$ (Blandford \& McKee 1976), $R_{\mathrm{s}} \propto t^{5 / 8}$, while for a wind profile with $k=2$ one gets $R_{\mathrm{s}} \propto t^{3 / 4}$. The predicted differences between the afterglow images in these two cases are typically small (Granot \& Loeb 2001). ${ }^{1}$

By resolving the image of a GRB afterglow at different frequencies, one can obtain precious, new constraints on the fireball model. As originally pointed out by Loeb \& Perna (1998), after about one day, the angular size of a GRB ring is typically a micro-arcsecond ( $\mu \mathrm{as})$, i.e. of the same order as the angular Einstein ring radius of a solar mass lens at cosmological distances,

$$
\theta_{\mathrm{E}}=\left(\frac{4 G M}{c^{2} D}\right)^{1 / 2}=1.6\left(\frac{M}{M_{\odot}}\right)^{1 / 2}\left(\frac{D}{10^{28} \mathrm{~cm}}\right)^{-1 / 2} \mu \mathrm{as}
$$

where $M$ is the lens mass, and $D \equiv D_{o s} D_{o l} / D_{l s}$, and $D_{o s}, D_{o l}$ and $D_{l s}$ are the angular diameter distances between the observer-source, observer-lens, and lens-source, respectively. This fortuitous coincidence, along with the fact that the afterglow image expands superluminally, means that lensing by an intervening solar-mass star will produce a detectable deviation in the afterglow light curve with a duration suitable for intense monitoring. As the afterglow image expands, different parts of it sweep by the lens and get amplified. The differential magnification of the source image can be used to recover the radial structure of the afterglow image through a series of relative flux measurements (Loeb \& Perna 1998; Mao \& Loeb 2001; Granot \& Loeb 2001). The probability that a source at redshift $z \sim 2$ will have a projected angular distance less than the Einstein radius of any intervening star (the optical depth) is $\tau \sim 0.3 \Omega_{*}$, where $\Omega_{*}$ is the cosmological density of stars in units of the critical density. The value of $\Omega_{*}$ is constrained to be $\gtrsim 1 \%$ based of the known populations of luminous (Fukugita, Hogan, \& Peebles 1998) and faint (Alcock et al. 2000) stars and $\lesssim 5 \%$ based on Big-Bang nucleosynthesis. Hence, $\tau \sim 1 \%$ (Press \& Gunn 1973; Blaes \& Webster 1992; Koopmans \& Wambsganss 2001), and roughly one out of a hundred afterglows should be strongly magnified (although all events may show weak magnification signals due to a star located at ten Einstein radii from their line-of-sight; see Mao \& Loeb 2001).

Intense monitoring of lensing events could provide invaluable information about the nature of GRB afterglows. Recently, Garnavich, Loeb \& Stanek (2000) interpreted the unusual optical afterglow light curve of GRB 000301C (Sagar et al. 2000; Rhoads \& Fruchter

\footnotetext{
${ }^{1}$ At very late times, of order months after the GRB trigger, the fireball becomes non-relativistic. In this regime, the observer sees the entire fireball which follows the Sedov-Taylor solution with $R_{\mathrm{s}} \propto t^{2 / 5}$.
} 
2000) as being microlensed by an intervening $\sim 0.5 M_{\odot}$ star. They found that the opticalinfrared light curve was well-fit by a model in which the emission originated from a ring of fractional width $\sim 10 \%$, in agreement with earlier theoretical predictions. Panaitescu (2001) argued that, if one adopts realistic surface brightness profiles for the afterglow image, the optical/infrared afterglow of GRB 000301C cannot be explained by microlensing. Gaudi, Granot, \& Loeb (2001) showed, however, that microlensing can reproduce the observed light curve, provided that the source is significantly limb-brightened, namely that $\gtrsim 60 \%$ of the flux arises from the outer $25 \%$ of the area of the afterglow. This requirement is met by the surface brightness profiles expected for emission frequencies above the cooling break frequency (Granot \& Loeb 2001). Unfortunately, the light curve coverage in radio frequencies was too sparse to uniquely confirm the microlensing interpretation through, e.g. comparison of the inferred surface brightness profiles across spectral breaks.

The idea of using microlensing to study the images of gamma-ray burst afterglows is similar in spirit to the idea of using microlensing to resolve the atmospheres of stars in the Magellenic clouds and the Galactic bulge (Loeb \& Sasselov 1995; Valls-Gabaud 1995; Sasselov 1997; Heyrovský, Sasselov, \& Loeb 2000); for a recent review, see Gould (2001). For background stars, the source radius stays constant and the resolution of the source occurs because the caustic structure of the lens sweeps over the face of the star due to the relative source-lens motion. However, for GRB afterglows the relative source-lens motion is negligible, and the resolution occurs as the image of the source itself is expanding and sweeping by the lens. Microlensing has been used to measure limb-darkening of the stars in the Small Magellenic Cloud and the Galactic bulge (Albrow et al. 1999a; Afonso et al. 2000; Albrow et al. 2000, 2001a) and also to measure the spatial variations of spectral lines across the face of two stars (Alcock et al. 1997; Castro et al. 2001; Albrow et al. 2001b; Heyrovský 2001). Gaudi \& Gould (1999) considered the signal-to-noise requirements for resolving stellar atmospheres with microlensing. Our goal here is to apply similar considerations to GRB afterglows.

In this paper, we examine the observational constraints that can be obtained on afterglow images through a concerted monitoring effort of the GRB community in future microlensing events. Specifically, we determine the expected statistical errors in the recovered intensity profile as a function of various parameters and input assumptions, assuming that the afterglow and microlensing event parameters are known. This significance of this work is twofold: first, we show that the radial intensity profile of afterglows can be determined with fairly good accuracy $(\lesssim 10 \%)$ for reasonable expense of observational resources, and second, we present the effects of various assumptions on the error in the recovered intensity profile, in order to test the robustness of the conclusions and to provide guidelines for observers. In $\S 2$ we present the formalism for microlensing of GRB afterglows. We describe the method 
for determining the expected errors in the recovered intensity profile in $\S 3$, and apply it to a fiducial case in $\S 4.1$. In $\S 4.2$, we determine the effects of various input assumptions on the errors. Finally, we summarize the implications of our primary results and discuss the effects of some of our (unavoidably) simplified assumptions in $\S 5$.

For concreteness, we will primarily focus on the scaling laws of a spherical fireball in a uniform medium, but derive a general scaling relation for the recovered errors in the relative intensity profile that is applicable for any power-law external density profile, $\rho \propto r^{-k}$. The two currently popular models, $k=0$ (uniform medium) and $k=2$ (stellar wind) yield qualitatively similar conclusions. We do not consider jetted outflows, although a collimated outflow of opening angle $\theta_{\text {jet }}$ would behave as if it is part of a spherical fireball at early time as long as $\Gamma(t)>1 / \theta_{\text {jet }}$ (Rhoads 1997). We also assume that the afterglow flux exhibits a single power-law slope, which is appropriate if the observed frequency does not cross one of the several breaks in the afterglow spectrum (see Granot \& Loeb 2001). Note that we do not assume any specific, model-dependent form for the intensity profile, i.e. we do not evaluate the errors on the coefficients of some parametric form for the profile. Rather, we evaluate the minimum attainable errors via direct inversion of the afterglow light curve.

\section{Gamma-Ray Burst Afterglows and Microlensing}

For a spherical fireball, the observer sees a circular source image with an outer radius of angular size $\theta$ that evolves as,

$$
\theta_{\mathrm{s}}(t)=\theta_{0} t_{\text {day }}^{\delta},
$$

where $\theta_{0}$ is the angular size of the afterglow after 1 day, $t_{\text {day }}$ is the observed time in days, and $\delta=[5-k] /[2(4-k)]$ for an external medium with density profile $\rho \propto r^{-k}$. The angular size $\theta_{0}$ depends on the energy of the burst, the density of the ambient medium, and the redshift of the burst. For typical parameters, $\theta_{0}$ is of order $1 \mu$ as. As long as an observed frequency, $\nu$, does not cross any of the time-dependent spectral break frequencies, the afterglow flux at that frequency evolves as a power-law of time (e.g., Sari et al. 1999)

$$
F_{\nu}(t)=F_{0, \nu} t_{\text {day }}^{-\alpha},
$$

where $F_{0, \nu}$ is the flux after 1 day. Granot \& Loeb (2001) provide a comprehensive study of the predicted image profile in the different spectral regimes.

Now assume that an intervening compact object lies at an angular distance $\theta_{b}$ from the line-of-sight to the center of the afterglow. We may then normalize the angular radius of the afterglow image in units of the Einstein radius of this object (Eq. 1),

$$
R_{\mathrm{s}}=R_{0} t_{\mathrm{day}}{ }^{\delta}
$$


where

$$
R_{0} \equiv \frac{\theta_{0}}{\theta_{\mathrm{E}}}
$$

Since both $\theta_{0}$ and $\theta_{\mathrm{E}}$ are $\mathcal{O}(1 \mu \mathrm{as})$ for typical parameters, $R_{0}$ is of order unity. The value of $R_{0}$ depends very weakly on the GRB energy output and the ambient density normalization, but is more sensitive to the lens mass and the source and lens redshifts (for $k=0$, see Eqs. $2 \& 3$ in Garnavich et al. 2000).

The magnification of an extended source is given by,

$$
\mu(t)=\frac{\int d^{2} r \mu_{0}(\mathbf{r}) I_{\nu}(t ; \mathbf{r})}{\int d^{2} r I_{\nu}(t ; \mathbf{r})}
$$

where $\mu_{0}(\mathbf{r})=\left(r^{2}+2\right) /\left(r \sqrt{r^{2}+4}\right)$ is the point-source magnification at vector position $\mathbf{r}$ relative to the lens, and the integral is over the area of the source. For lensing of a uniform circular source of radius $R_{\mathrm{s}}$ by a single point mass with no external shear, the magnification obtains the value $\mu\left(t ; R_{\mathrm{s}}, b\right)=\Psi\left[r=R_{\mathrm{s}}(t), b\right]$, where (Schneider, Ehlers \& Falco 1992; Witt \& Mao 1994),

$$
\Psi(r, b)=\frac{2}{\pi r^{2}}\left[\int_{|b-r|}^{b+r} d R \frac{R^{2}+2}{\sqrt{R^{2}+4}} \arccos \frac{b^{2}+R^{2}-r^{2}}{2 R b}+H(r-b) \frac{\pi}{2}(r-b) \sqrt{(r-b)^{2}+4}\right] .
$$

Here $H(x)$ is the step function and $b \equiv \theta_{b} / \theta_{E}$ is the angular separation (impact parameter) between the source center and the lens in units of the Einstein ring radius.

For a uniform source (as applicable below the synchrotron self-absorption frequency; see Granot et al. 1999b and Granot \& Loeb 2001), the magnification history by a point-mass lens is completely specified by two parameters: $b$ and $R_{0}$. Figure 1 shows the magnification as a function of time in days for $R_{0}=1$ and $b=1$, and two density profiles, uniform $(\delta=5 / 8)$ and $\rho \propto r^{-2}(\delta=3 / 4)$. At early times when the source radius is small, the magnification is roughly fixed at its point-source value $\Psi_{0}(b)=\mu_{0}(b)=\left(b^{2}+2\right) /\left(b \sqrt{b^{2}+4}\right)$. The peak magnification occurs at a time $t_{\text {peak }}$ when $R\left(t_{\text {peak }}\right)=b$, i.e. $t_{\text {peak }} \approx\left(b / R_{0}\right)^{1 / \delta}$ days. The total flux from the afterglow is then by,

$$
F_{\text {tot }, \nu}(t)=F_{0, \nu} \Psi(t ; R, b) t_{\text {day }}{ }^{-\alpha}+F_{\text {bg }},
$$

where we have allowed for flux $F_{\text {bg }}$ from any unresolved sources not being lensed, e.g. the host galaxy of the GRB. Thus, in the simplest scenario the flux at a given frequency is a function of five parameters: $F_{0, \nu}, \alpha, F_{\mathrm{bg}}, R_{0}$, and $b$.

In order to illustrate how microlensing effectively resolves the image of the GRB afterglow, we plot in Figure 1 the fraction of $F_{\text {tot }}$ contributed by five equal area annuli as 
a function of time assuming $b=1, R_{0}=1$, and a uniform source. For $t \lesssim t_{\text {peak }}$, the annuli contribute roughly equal flux, and the source is not resolved. However, beginning at $t \sim t_{\text {peak }}$ different annuli obtain different weights and the lens differentially magnifies the source. The annulus that contributes most of the flux at a given time has a radius of $r_{\text {peak }}=r_{0} t^{\delta} \sim b$ in units of $\theta_{\mathrm{E}}$, where $r_{0}$ is the radius of that annulus at $t=1$ day. Defining the fractional radius $X \equiv r / R_{\mathrm{s}} \equiv r_{0} / R_{0}$,

$$
X_{\text {peak }}=\left(\frac{t}{t_{\text {peak }}}\right)^{-\delta} .
$$

From inspection of Figure 1 and equation (9), it is clear that the the light curve need only be monitored from $t_{\text {peak }}$ until $0.3^{-1 / \delta} t_{\text {peak }}$ to resolve the outer $70 \%$ of the radial profile of the afterglow image.

\section{Error Analysis}

Consider an azimuthally symmetric afterglow divided into $N_{\mathrm{r}}$ annuli, with annulus $i$ centered at radius $r_{i}$. We assume a self-similar behavior for which both the fractional radii $X_{i}$ and the mean relative intensity $\mathcal{I}\left(r_{i}\right) \equiv I\left(t ; r_{i}\right) / I(t)$ in each annulus $i$ are constants in time. The total flux from the afterglow is simply the sum of the fluxes from each annulus. The weight of each annulus is in turn the area of the image of each annulus of width $\Delta r_{i}$,

$$
\Omega\left(t ; r_{i}\right)=\pi\left[\Psi\left(t ; r_{i}+\Delta r_{i}\right)\left(r_{i}+\Delta r_{i}\right)^{2}-\Psi\left(t ; r_{i}-\Delta r_{i}\right)\left(r_{i}-\Delta r_{i}\right)^{2}\right]
$$

times the mean intensity of that annulus. Converting equation (6) from an integral to a finite sum, the total flux is simply,

$$
F_{\text {tot }}(t)=F_{\text {bg }}+I(t) \sum_{i}^{N_{\mathrm{r}}} \Omega\left(t ; r_{i}\right) \mathcal{I}\left(r_{i}\right) .
$$

The coefficients one wishes to determine are the $N_{r}$ relative intensities $\mathcal{I}\left(r_{i}\right)$. These can be determined through inversion of the observed flux $F_{\text {tot }}(t)$ if $F_{\mathrm{bg}}, I(t)$, and $\Omega\left(t ; r_{i}\right)$ are known. The functions $I(t)$ and $\Omega\left(t ; r_{i}\right)$ in turn depend on the parameters $F_{0, \nu}, \alpha, b$, and $R_{0}$. Schematically, the inversion can be done as follows: $\alpha$ and $F_{\text {bg }}$ can be determined from the late-time $\left(t \gg t_{\text {peak }}\right)$ behavior of the afterglow light curve, when the magnification due to microlensing is negligible. The unlensed flux of the afterglow could then be extrapolated back to $t=1$ day, to determine $F_{0, \nu}$. The offset between the lensed and unlensed flux at early times $t \ll t_{\text {peak }}$ then gives $b$. Finally, a measurement of $b$ combined with $t_{\text {peak }}$ provides $R_{0}$. Therefore, all the parameters necessary to invert equation (11) can ideally be determined without reference to the surface brightness distribution of the source image. 
In practice, of course, a global fit to all $5+N_{r}$ parameters must be done simultaneously, and this will introduce correlations between the parameters $\mathcal{I}\left(r_{i}\right)$ and $F_{0, \nu}, \alpha, F_{\mathrm{bg}}, R_{0}, b$, in turn inflating the errors on the measured values of $\mathcal{I}\left(r_{i}\right)$. While $F_{\text {tot }}$ is linearly dependent on $\mathcal{I}\left(r_{i}\right)$ (see Eq. 11), this is not true for the parameters $F_{0, \nu}, \alpha, F_{\mathrm{bg}}, R_{0}, b$. The nonlinear dependences of these parameters on the observed quantity $F_{\text {tot }}$ make the expected errors on these parameters and their covariances with the parameters of interest, $\mathcal{I}\left(r_{i}\right)$, extremely difficult and time consuming to calculate. Furthermore, the errors will depend sensitively on the exact coverage and accuracy of the afterglow photometry. For an observed microlensing event, a full and careful determination of the errors on the inferred values of $\mathcal{I}\left(r_{i}\right)$ must account for their covariances with the other fit parameters. However, including these effects here is beyond the scope of the paper. Therefore, when computing the error bars on $\mathcal{I}\left(r_{i}\right)$, we will for simplicity assume that $F_{0, \nu}, \alpha, F_{\mathrm{bg}}, R_{0}, b$ are perfectly known. We stress that the errors we derive should thus be regarded as lower limits to the actual errors.

Now consider a series of flux measurements $F_{\text {tot }}\left(t_{k}\right)$ that are made at times $t_{k}$ with errors $\sigma_{k}$, and that are fit to equation (11). The parameters of the fit are $\mathcal{I}_{i} \equiv \mathcal{I}\left(r_{i}\right)$, and the variances in these parameters are $\delta \mathcal{I}_{i}=\left(\mathcal{C}_{i i}\right)^{1 / 2}$, where $\mathcal{C}_{i j}$ is the covariance matrix,

$$
\mathcal{C}=\mathcal{B}^{-1}, \quad \mathcal{B}_{i j}=\sum_{k} \sigma_{k}^{-2} \frac{\partial F\left(t_{k}\right)}{\partial \mathcal{I}_{i}} \frac{\partial F\left(t_{k}\right)}{\partial \mathcal{I}_{j}},
$$

and $\partial F\left(t_{k}\right) / \partial \mathcal{I}_{i}=I\left(t_{k}\right) \Omega\left(t ; r_{i}\right)$. The fractional errors are then $\delta \mathcal{I}_{i} / \mathcal{I}_{i}=\mathcal{C}_{i i}^{1 / 2} / \mathcal{I}_{i}$. We assume Poisson-noise limited precision, so that $\sigma_{k} \propto \sqrt{F\left(t_{k}\right)}$. Therefore, for a given set of assumptions about the microlensing event $\left(b, R_{0}\right)$, the unlensed flux of the afterglow $\left(F_{0, \nu}, F_{\mathrm{bg}}\right)$ and the observational setup (duration of observations, telescope diameter, etc.), the covariance matrix $\mathcal{C}$ can be formed and the expected errors $\delta \mathcal{I}_{i} / \mathcal{I}_{i}$ determined.

\section{Results}

\subsection{Fiducial Scaling Relation}

We now apply the formalism in $\S 3$ to derive an approximate scaling relation for $\delta \mathcal{I}_{i} / \mathcal{I}_{i}$. Rather than adopt specific (and therefore model dependent) forms for the afterglow flux $F_{0, \nu}$ as a function of frequency and a specific observational setup, we will simply assume that the instrument being used collects $\Gamma_{\nu}$ photons per second from the unlensed afterglow at $t=1$ day. In $\S 4.5$, we will evaluate values of $\Gamma_{\nu}$ and thus the errors expected from a specific example of afterglow emission and observational setup. For our fiducial scenario, we assume a uniform source $\left(\mathcal{I}_{i}=1\right)$, a negligible background flux $\left(F_{\mathrm{bg}}=0\right)$, and that observations are made continuously from the burst until very late times $t \gg t_{\text {peak }}$. We also assume equal-area 
annuli, so that all bins have equal weight for a uniform source. We then calculate $\delta \mathcal{I}_{i} / \mathcal{I}_{i}$ for a range of values of $\alpha, R_{0}, b$, and $N_{r}$. Numerically, we find the following approximate scaling relation for the fractional errors in the recovered intensity profile,

$$
\left(\frac{\delta \mathcal{I}_{i}}{\mathcal{I}_{i}}\right)_{0}=0.35\left(\frac{r_{i}}{R_{s}}\right)^{5(1-\alpha) / 8 \delta+3 / 2}\left(\frac{\Gamma_{\nu}}{1 \mathrm{~s}^{-1}}\right)^{-1 / 2}\left(\frac{N_{r}}{10}\right)^{8 / 5} R_{0}^{(1-\alpha) / 2 \delta} b^{\alpha / 2 \delta} .
$$

Equation (13) is the primary result of this paper. We find that it predicts the expected errors to an accuracy of $\lesssim 5 \%$ for most combinations of parameters. Note that equation (13), and indeed the majority of the quantitative results in this paper, rely on four assumptions about the afterglow, namely: (i) the unlensed flux of the afterglow is a power-law function of time, $F \propto t^{-\alpha}$; (ii) the radius of the afterglow image scales as $R_{\mathrm{s}} \propto t^{\delta}$; (iii) the image profile evolves self-similarly; and (iv) the afterglow image is circularly symmetric. These assumptions should hold at least approximately for some restricted part of most afterglow light curves. We discuss these assumptions more thoroughly in $§ 5$. The above equations can be used to determine the expected errors on the recovered intensity from observations performed with various instruments, apertures, and photon frequencies of afterglow light curves of arbitrary fluxes and power-law indices. Because we have assumed that the observational precision is limited only by photon statistics, that there are no correlations with the other parameters (see the discussion in $\S 3$ ), and that the measurements are continuous, equation (13) provides the minimum attainable error, at least for uniform sources and isolated lenses with no external shear. In the next section, we will relax some of the assumptions leading to this result in order to evaluate their effect on the expected errors. For the two cases of a uniform medium $(\delta=5 / 8)$ and a stellar wind medium $(\delta=3 / 4)$, the exponents in equation (13) are quite similar. Therefore, for the sake of simplicity, we will consider only the uniform medium case with $\delta=5 / 8$, in the discussion that follows.

Several of the terms in equation (13) can be derived analytically, simply by noting that, according to photon statistics, the errors should scale as $\delta \mathcal{I}_{i} / \mathcal{I}_{i} \propto N_{\gamma}^{-1 / 2}$, where $N_{\gamma}$ is the number of photons collected. For example, the scaling with $R_{0}$ can be derived as follows: the time of the peak of the light curve scales as $t_{\text {peak }} \propto R_{0}^{1 / \delta}$. The unlensed flux at this time is $F\left(t_{\text {peak }}\right) \propto R_{0}^{-\alpha / \delta}$. The magnification structure is independent of $R_{0}$; however, all times are scaled by $R_{0}^{1 / \delta}$, so the time over which photons can be collected scales as this factor. Thus the total error should scale as $\left(R_{0}^{1 / \delta} R_{0}^{-\alpha / \delta}\right)^{1 / 2}=R_{0}^{(1-\alpha) / 2 \delta}$, as found numerically. The scaling with the impact parameter $b$ can be derived in a similar fashion, under the assumption that the peak magnification scales as $b^{-1}$. However, this is only strictly valid for $b \ll 1$. Therefore, the scaling $\delta \mathcal{I}_{i} / \mathcal{I}_{i} \propto b^{\alpha / 2 \delta}$ in equation (13) is only approximate, and breaks down for $b \gg 1$. Numerically, we find that for $b \leq 4$, equation (13) predicts errors that are too small by $\lesssim 20 \%$ for $r \gtrsim 0.5 R_{s}$ (the outer $70 \%$ of the area of the image). The exponent for the scaling with $N_{r}$ is 8/5, similar to that found by Gaudi \& Gould (1999) for resolving the images 
of Galactic stars by microlensing. Naively, one might expect the prefactor in equation (13) to be considerably smaller: for $\alpha=1$ and $b=1$ the total number of photons collected is of order $N_{\gamma} \sim 10^{5}\left(\Gamma_{\nu} / 1 \mathrm{~s}^{-1}\right.$ ) (with only a logarithmic dependence on the integration time during which the source is resolved). Divided over $N_{r}=10$ bins, one might expect the error per bin to be $\left(N_{\gamma} / N_{r}\right)^{-1 / 2} \sim 1 \%\left(\Gamma_{\nu} / 1 \mathrm{~s}^{-1}\right)^{-1 / 2}$, about an order of magnitude smaller than the $\sim 12 \%\left(\Gamma_{\nu} / 1 \mathrm{~s}^{-1}\right)^{-1 / 2}$ predicted by equation (13) for a radius $r_{i}=0.5 R_{s}$. However, the errors increase with $N_{r}$ faster than one would naively expect, and so the error in each bin is closer to $\left(N_{\gamma} / N_{r}^{16 / 5}\right)^{-1 / 2} \sim 12 \%\left(\Gamma_{\nu} / 1 \mathrm{~s}^{-1}\right)^{-1 / 2}$.

\subsection{Effects of Changes in the Input Assumptions}

\subsubsection{Duration of Observations}

The most severe simplification made in deriving equation (13) is the assumption of continuous measurement from the GRB trigger until long after the peak time $t_{\text {peak }}$. Since the optical depth for microlensing is $\tau \sim 1 \% b^{2}$, at least $100 b^{-2}$ afterglows must be monitored to detect one that has impact parameter $\leq b$. If it was truly necessary to monitor all of these afterglows continuously to recover the intensity profile accurately, this would represent an overwhelming observational burden. We therefore evaluate the errors expected under various assumptions about the starting time and the duration of the observations. We scale the starting time, $\tau_{\text {start }}$, and ending time, $\tau_{\text {end }}$, in terms of the peak time through the relation $\tau \equiv\left(t / t_{\text {peak }}\right)^{5 / 8}$, so that for given $\tau_{\text {start }}$ and $\tau_{\text {end }}$, a fixed range of fractional radii, namely $X_{\min }=\tau_{\text {end }}^{-1}$ through $X_{\max }=\tau_{\text {start }}^{-1}$, are probed regardless of the values of $R_{0}$ and $b$ (see Eq. 9). The top panel of Figure 2 shows the expected errors $\delta \mathcal{I}_{i} / \mathcal{I}_{i}$ normalized to the fiducial errors in equation $(13)$, for $\left(\tau_{\text {start }}, \tau_{\text {end }}\right)=(0.1,100.0)$, corresponding to complete coverage of the microlensing event, and also for $\tau_{\text {start }}=1$ (starting at the peak of the event) and various values of $\tau_{\text {end }}$. Note that here and throughout we plot $\delta \mathcal{I}_{i} / \mathcal{I}_{i}$ as a function of $\left(r / R_{s}\right)^{2}$, because we assume bins of equal area (and hence equal weight for a uniform source; see Eq. 10). Clearly observations before the peak of the event provide little information on the intensity profile. Moreover, the afterglow need only be monitored until $t \sim 7 t_{\text {peak }}$ to resolve the outer two thirds of the source radius. The bottom panel of Figure 2 shows the expected errors when $\tau_{\text {start }}$ is varied. Delaying observations even somewhat past the peak of the event will result in seriously degraded information.

We conclude that measurements from $t_{\text {peak }}$ until $\sim 7 t_{\text {peak }}$ will provide almost as much information as continuous measurements from the GRB trigger until late times. Since for typical parameters $t_{\text {peak }} \sim 1$ day, the afterglow need only be aggressively monitored for about six days. This suggests the following detection strategy for maximizing the number of 
lensed GRBs for which the intensity profile can be recovered. Since the sampling necessary to determine that the GRB is being microlensed is not as dense as that needed to recover the intensity profile, a large number of bursts can be monitored relatively infrequently from a global network of small telescopes. With real-time reduction, this network should be able to "alert" microlensing events before or near the peak time. Larger telescopes could then be used to densely sample the microlensed afterglow for the $\sim 6$ days necessary to resolve the intensity profile. This is similar to the way Galactic microlensing observations are coordinated (Udalski et al. 1994; Alcock et al. 1996).

\subsubsection{Realistic Intensity Profiles}

In this section, we explore the effects of realistic intensity profiles on the resultant errors. The optical image of the afterglow is expected to be limb-brightened, because light from the edge of the afterglow suffers the longest time delay, and thus was emitted at earlier times when the fireball was brighter (Waxman 1997c; Sari 1998; Panaitescu \& Meszaros 1998; Granot, Piran, \& Sari 1999a,b). The contrast between the center and edge of the afterglow, as well as the sharpness of the cut-off in intensity at the outer edge of the afterglow, depends on the location of the observed frequency relative to the various spectral breaks in the broken power-law spectrum of afterglows (Granot et al. 1999a,b; Granot \& Loeb 2001). The exact shape of the relative intensity profile will affect the shape of the light curve, as well as the resultant errors, with the errors expected to be larger near the center (where the relative intensity is smaller) than those calculated assuming a uniform source. Previous studies of microlensing (Loeb \& Perna 1998; Garnavich, Loeb \& Stanek 2000; Mao \& Loeb 2001) have parameterized the intensity profile as a uniform inner disk bounded by a uniform outer ring, with two parameters specifying the width $W$ and contrast $C$ of the ring. Of course, realistic intensity profiles do not exhibit such discontinuous features. The precise calculation of these profiles for the different spectral regimes of GRB afterglows is presented elsewhere (Granot \& Loeb 2001). Here, we adopt a parameterized form for the relative intensity profile that captures the qualitative aspects of realistic profiles,

$$
\mathcal{I}(X)=\mathcal{I}_{0}\left[1-c \sqrt{1-X^{2}}\right]\left(1-X^{n}\right),
$$

where $c$ and $n$ are free parameters that define the shape of the profile, and $\mathcal{I}_{0}$ is the normalization such that $\int_{0}^{1} X d X \mathcal{I}(X)=1$. The index $n$ defines the sharpness of the cut-off, and is roughly analogous to the width of the ring $W$. The coefficient $c$ is related to the intensity at $X=0$, and is roughly analogous to the contrast $C$. Note that for $c=0$ and $n \rightarrow \infty$, this form reduces to a uniform source. 
The top panel of Figure 3 shows $\mathcal{I}(X)$ for a relatively gradual cutoff, $n=20$, and various values of $c$. The bottom panel of Figure 3 shows the fractional errors $\delta \mathcal{I} / \mathcal{I}_{0}$ relative to the fiducial errors for a uniform source in equation (13), given the image profiles shown in the top panel. Note that we normalize the variances $\delta \mathcal{I}$ to the mean intensity $\mathcal{I}_{0}$ rather than the intensity in that bin in order to avoid the fractional errors $\delta \mathcal{I} / \mathcal{I}$ blowing up when $\mathcal{I} \rightarrow 0$ for large $c$. In general, the resultant errors are a factor $\lesssim 3$ worse than than the uniform source case. In the most extreme example where $\mathcal{I}(X) \rightarrow 0$ at the center of the image, the fractional errors can become quite large, but only for the innermost bin. For the outer half of the area of the image, the errors can be smaller than the uniform source case. Thus, in this case, measurements will result in a robust upper limit to the intensity at the center of the image, with a clear measurement for the outer part of the image. Therefore the ring-like structure of the image will be accurately recovered. We have also calculated, but do not show, the errors for the case of a sharp outer cutoff, $n=500$. The results for this case are qualitatively and quantitatively similar to the results for $n=20$. We conclude that realistic intensity profiles will not result in significantly inflated errors relative to the uniform source estimate (Eq. 13), at least for the majority of the source area.

\subsection{External Shear and Binary Lenses}

We now consider more complicated models for the lens. The majority of lenses are likely to reside in galaxies, and a significant fraction of these may be in binary systems. Therefore, the assumption of an isolated single lens with no external shear used in the previous sections will not be valid. We therefore consider two other types of lenses: a single lens with external shear (Chang \& Refsdal 1979), and a binary lens (Schneider \& Weiss 1986). The former case is appropriate for a lens perturbed by either a wide binary companion or the potential of its host galaxy. The latter is appropriate for binary-lenses with separations $\mathcal{O}\left(\theta_{\mathrm{E}}\right)$ or smaller. For a lens residing in a region of high optical depth, i.e. near the center of a galaxy, the magnification structure will not be well approximated as either an isolated lens with external shear or a binary lens. In this case one may only be able to draw statistical inferences about the surface brightness profile of the source, as it will be difficult to reconstruct the magnification structure of the lens from the light curve alone. We will not consider this regime here. We compute the fractional errors in the recovered intensity profiles in the same manner as before, except that we must replace $\Psi$ in equation (10) with the uniform-source magnification appropriate for the given lens model. We compute this magnification using the inverse ray-shooting method (see, e.g., Wambsganss 1997) as follows. The generalized

lens equation for point masses and external shear can be expressed in complex coordinates 
on the plane of the sky as (Witt 1990),

$$
\zeta=\gamma \bar{z}+z+\sum_{k}^{N} \frac{\epsilon_{k}}{\bar{z}_{k}-\bar{z}}
$$

where $\zeta$ is the complex source position, $z$ is the complex image position, $\gamma$ is the shear, $\epsilon_{k}$ is the fractional mass of lens component $k$, and $z_{k}$ is the position of this component. All distances are expressed in units of the angular Einstein ring of the combined mass of the system. For a single lens, $N=1, \epsilon_{1}=1$, and $z_{1}=0$. For a binary-lens with no external shear, $\gamma=0, N=2$, the mass ratio is $q=\epsilon_{1} / \epsilon_{2}$, the dimensionless projected separation is $d=\left|z_{1}-z_{2}\right|$, and we choose the origin to be the midpoint of the binary.

We sample the image plane uniformly and densely, solving for the source position using the appropriate form of equation (15). These positions are then binned in the source plane. The magnification of each bin is then just the surface density of rays in the image plane divided by the surface density of rays in the source plane. Since this procedure conserves flux, the resulting magnification map can then be convolved with a source of arbitrary size to compute the magnification of the extended source. We calculate magnification maps for $\gamma=0.1,0.2,0.3$ and 0.4 , and one binary-lens configuration with $d=0.8$ and $q=1$. For the single lens with external shear, $N_{r}+7$ parameters are needed to specify the light curve, namely: the $N_{\mathrm{r}}$ relative intensities $\mathcal{I}_{i}$, plus $F_{0, \nu}, \alpha, F_{\mathrm{bg}}, R_{0}$, the shear $\gamma$, and two parameters that specify the position of the GRB with respect to the lens, which we denote as $(x, y)$. For the binary lens, we assume that $\gamma=0$; however two additional parameters are needed to describe the topology of the lens, namely $d$ and $q$, and so there are a total of $N_{r}+8$ parameters. When calculating the errors on the parameters $\mathcal{I}_{i}$, we will again assume that all other parameters are perfectly known. Here this assumption is somewhat less justified than the single lens case, not only because there are more parameters, but also because the effects of these parameters are in general not directly related to features in the light curve, and are likely to be subject to degeneracies or correlations, which will inflate the errors in the recovered $\mathcal{I}_{i}$. Such denegeracies or correlations are known to inflate the errors in limbdarkening measurements of stars using Galactic binary-lens events, although not greatly so for well-measured light curves (Afonso et al. 2000; Albrow et al. 2001a). In the Galactic binary-lens case, determination of all parameter combinations that correspond to solutions to the observed light curve, and therefore identification of degeneracies and correlations between fit parameters, is a formidable task (Mao \& di Stefano 1995; di Stefano \& Perna 1997; Albrow et al. 1999b). This is likely to also be true for GRB afterglows. A comprehensive study of parameter space is beyond the scope of this paper, and so we will simply assume that the binary lens parameters are known. This assumption facilitates comparison with the isolated single-lens case, which is the main purpose of our discussion here. We stress that the errors 
we derive under this assumption are almost certainly underestimates. In both the external shear and binary-lens case, we will assume a uniform afterglow image (see Mao \& Loeb 2000 for other examples).

In Figure 4 we show the magnification light curves for the case of a single lens with external shear along the $x$-axis of magnitudes $\gamma=0,0.1,0.2,0.3$, and 0.4 , both for a source centered on the x-axis of the lens, $(x, y)=(1,0)$, and a source centered on the $\mathrm{y}$-axis of the lens $(x, y)=(0,1)$. Note that the caustics are most highly elongated along the $\mathrm{x}$-axis, and thus for a given $\gamma$, light curves where the source is centered on the $\mathrm{x}$-axis should show more dramatic deviations from the $\gamma=0$ case than for sources centered on the y-axis (Mao \& Loeb 2000). Figure 5 shows the resultant errors relative to the fiducial error estimate in equation(13). In general, sources centered on the x-axis show more dramatic variations in the expected errors than sources centered on the y-axis. In all cases the fractional errors are within a factor of 2 of the $\gamma=0$ expectation. Thus, at least for modest values of $\gamma \leq 0.4$, we conclude that an external shear will not result in significantly inflated statistical errors, assuming that the covariances with the afterglow and microlensing parameters are small.

In Figure 6, we show the magnification as a function of time for the binary lens with $d=0.8$ and $q=1$, and a source centered at $(x, y)=(-0.16,-1)$. We also show the fraction of total flux contributed by five equal area bins as a function of time. For times $t>1$ day, the light curves resemble that for a single lens (compare with Figure 1), as the annuli become comparable to or larger than the caustic features. The bottom panel of Figure 6 shows the resultant errors relative to the fiducial single-lens case. Again, the statistical errors differ by less than a factor of 2 from the fiducial estimate in equation (13).

\subsection{Finite Unresolved Flux}

Observed afterglows often show evidence for a flattening of the light curve to a constant flux at late times. This is usually interpreted as due to unresolved flux from the host galaxy of the GRB. Even if the flux from the host galaxy is small, the sky background will eventually dominate over that of the afterglow. In other words, it is inevitable that $F_{\nu}(t)<F_{\mathrm{bg}}$ at late times. For ground-based infrared observations, the background flux is likely to be larger than the afterglow flux at all relevant times. A finite background flux will increase the errors on the recovered parameters $\mathcal{I}_{i}$, and preferentially so for smaller radii, since the information on smaller radii comes at later times, when the background is more significant. We explore this effect by introducing a background flux which amounts to some fraction of $F_{\nu, 0}$ for the afterglow. Figure 7 shows the expected fractional errors on the recovered intensity

profile relative to the fiducial value in equation (13), assuming a power-law flux index of 
$\alpha=1$ for the afterglow, for relative background fluxes of $F_{\mathrm{bg}} / F_{\nu, 0}=0.01,0.1,0.4,1.0,2.5$, and 10.0. At optical-infrared frequencies, this corresponds to a background of magnitude $5,2.5,1,0,-1,-2.5$ relative to the (unlensed) afterglow at 1 day. As expected, the errors generally increase as the background flux increases, especially for smaller radii. However, unless the background flux is higher than $F_{\nu, 0}$, the expected errors are $\lesssim 3$ times greater than the fiducial estimate. In other words, for optical frequencies, the background flux should not compromise the measurement of the intensity profile. However, for, e.g., groundbased observations in the near-infrared, where the sky background can be many magnitudes brighter than the afterglow flux at 1 day, the accuracy with which the intensity profile can be measured will considerably larger than the fiducial estimate (Eq. 13). Thus spaced-based near-infrared observations would improve the accuracy of the recovered intensity profile.

\subsection{A Worked Example}

As discussed in $\S 4.1$, the results we have presented so far are quite general. We now estimate the errors expected under realistic observational conditions and a realistic model. To do so, we make estimates of the various terms in equation (13) by adopting the parameters for the observed afterglow of GRB 000301C. Garnavich, Loeb \& Stanek (2000) fit the optical and infrared photometry of GRB 000301C to double power-law flux decline magnified by the simple microlensing model of Loeb \& Perna (1998). They find best-fit parameters of $\alpha_{1}=1.1$ and $\alpha_{2}=2.9$ for the power-law flux indices, similar to the values found originally by Sagar et al. (2000) without including the microlensing modification. For simplicity, we ignore the break, and simply adopt a single power-law of slope $\alpha=1$. . We adopt the bestfit microlensing parameters for the entire dataset of $R_{0}=0.49$ and $b=1.04^{2}$. Finally, we extrapolate by eye the unlensed flux of the afterglow back to $t=1$ day for the $U, B, V, R, I, J$ and $K$-bands. Table 1 shows the demagnified, extrapolated magnitudes at $t=1$ day of the afterglow of GRB $000301 \mathrm{C}$, along with the assumed sky brightness, and $\Gamma_{\nu} / A_{\mathrm{T}}$, the number of photons per second per square meter of collecting telescope area $A_{\mathrm{T}}$. Note that, for $R_{0}=0.49$ and $b=1.04$, the peak of the microlensing event (for a uniform source) occurs at $t_{\text {peak }}=\left(b / R_{0}\right)^{8 / 5} \approx 3$ days. We calculate the expected errors $\delta \mathcal{I} / \mathcal{I}$ assuming a $4-\mathrm{m}$ telescope, a seeing of $1^{\prime \prime}$, a uniform source, and observations between the peak, $t=t_{\text {peak }}=$ 3 days and $t \simeq 9 t_{\text {peak }} \simeq 30$ days. We adopt a uniform source in order to provide a modelindependent estimate of the fractional errors expected for realistic observing conditions.

\footnotetext{
${ }^{2}$ Gaudi, Granot, \& Loeb (2001) find the impact parameter in GRB 000301C is not well-constrained, but is in the range $0.2 \lesssim b \lesssim 0.7$, somewhat smaller than that found by Garnavich, Loeb \& Stanek (2000). For illustrative purposes, we will simply adopt the Garnavich, Loeb \& Stanek (2000) value of $b=1.04$.
} 
Image profiles in the optical and infrared are unlikely to be well-represented by a uniform source (Granot \& Loeb 2001), however this will not affect the errors in the recovered profile by more than a factor of $\sim 3$ (see $\S 4.2 .2)$. The results are shown in Figure 8. For observations in the optical, the relative intensity profile can be recovered accurately, $\delta \mathcal{I} / \mathcal{I} \lesssim 3 \%$. For observations in the infrared, where the sky background is dominant, the expected errors are $\sim 10 \%$. Although we do not specifically consider other wavelengths here, we note that radio afterglows peak later and last longer than afterglows at shorter wavelengths (Kulkarni et al. 2000 and references therein). This, combined with the lower energy of the photons, implies that the photon flux at a given time is larger in the radio than in the optical. At sufficiently high radio frequencies where the effect of scintillations is sub-dominant, radio observations may provide more accurate measurements of the afterglow image. Since the afterglow image is expected to be different at radio frequencies (Granot \& Loeb 2001), we advocate performing measurements over as wide a range of frequencies as possible.

Given that the fraction of afterglows that exhibit microlensing events with impact parameter $\leq b$ scales as $b^{2}$, it is interesting to consider what kind of errors one would expect for the specific example of GRB 000301C considered above, but with larger impact parameters. We therefore repeat the calculation above with the same parameters, except we now vary the impact parameter. Specifically, we consider $b=1,2,3,4$, and 5 , as would be expected in $\sim 1 \%, 4 \%, 9 \%, 16 \%$, and $25 \%$ of all GRB afterglows. In Figure 9 we show the results for the $U$-band (where the background is the smallest), assuming that observations are taken from peak, $t=t_{\text {peak }}=3 b^{5 / 8}$ days until $t \simeq 9 t_{\text {peak }} \simeq 30 b^{5 / 8}$ days.

For $b \leq 3$, the fractional errors in the recovered intensity profile are $\lesssim 10 \%$ for the majority of the area of the image. Thus, it should be possible to recover the intensity profile to an accuracy of $\lesssim 20 \%$ for $\sim 10 \%$ of all afterglows. To test the robustness of this conclusion, we also show inFigure 9 the results for the $b=3$ case, but considering a band with larger background flux $(R$-band), and also a non-zero external shear $(\gamma=0.2$ with source on the shear axis). We find that the error is not greatly inflated, $\delta \mathcal{I} / \mathcal{I} \lesssim 20 \%$. Of course, these results assume that the afterglow can be monitored for many months after the peak of the microlensing event. Practical aspects aside, such monitoring may not be possible even in principle in those cases where the emission is due to a highly collimated jet. Such afterglows will eventually exhibit breaks in the light curve and changes in the image structure when the Lorentz factor of the fireball falls below the inverse of the opening angle of the jet. In this case, the errors on the annuli centered at smaller radii would be inflated (see §4.2.1). Additional deviations from our results may occur as the fireball decelerates to non-relativistic speeds (Mao \& Loeb 2001). 


\section{Conclusions}

Microlensing offers a unique method for probing the dynamics of GRB fireballs. As the GRB afterglow image expands, it is differentially magnified by the lens. Different annuli of the image are resolved as they cross the lens position at different times. Thus the light curve of the afterglow during the microlensing event can be inverted to obtain the radial intensity profile $\mathcal{I}(X)$, where $X=r / R_{\mathrm{s}}$ is the fractional radius of the circular image. We developed the formalism necessary to calculate the expected fractional errors on the recovered intensity profile $\delta \mathcal{I} / \mathcal{I}$.

Assuming continuous observations, uniform sources, single lenses, and zero background flux, we find that the expected errors follow a general scaling relation (Eq. 13) in terms of the afterglow flux, afterglow radius in units of the Einstein radius, the angular separation between the afterglow and the lens, and the number of resolution elements in the recovered intensity profile. We have tested the accuracy of this relation by, in turn, relaxing the assumptions of continuous observations, uniform sources, single lenses, and zero background flux. We find that, for reasonable choices of the relevant parameters, none of these refinements changes the expected errors by more than a factor of $\sim 3$. Therefore, the fiducial scaling relation is fairly robust.

Notably, we have found that observations starting at the peak $t_{\text {peak }}$ of the microlensing event and ending at $7 t_{\text {peak }}$ result in almost the same accuracy as measurements of the entire afterglow. Therefore, one can monitor frequently only those afterglows that are significantly microlensed by "alerting" to these events before they reach their peak.

Finally, we calculated the errors expected for observations in the near-infrared and optical regimes of a specific example. Adopting the observed and inferred parameters for the (possibly) microlensed optical/infrared afterglow of GRB 000301C, we find that the relative intensity profile can typically be measured with a resolution of 10 and accuracy of $\mathcal{O}(1 \%)$ in the optical and $\mathcal{O}(10 \%)$ in the infrared using 4m-class telescopes. Fluctuations in the light curve due to inhomogeneities in the ambient gas distribution are typically small and should not compromise this precision (Wang \& Loeb 2000; Halpern et al. 2000).

In the course of this study, we have had to make a number of simplifying assumptions. These include: (i) the unlensed flux of the afterglow is a power-law function of time, (ii) the radius of the afterglow image scales as a power-law of time $R_{\mathrm{s}} \propto t^{\delta}$, (iii) the afterglow image is circularly symmetric, (iv) the intensity profile is self-similar, i.e. $\mathcal{I}(X)$ is independent of time, (v) the lens resides in a region of relatively low optical depth, and (vi) the covariances between

the surface brightness profile parameters and the afterglow and microlensing parameters are small. The first assumption is a generic prediction of the simplest afterglow models, 
provided that the observed frequency does not cross one of the several spectral breaks, and that $1 / \Gamma$ is smaller than the collimation angle of the ejecta. When a break in the light curve occurs - either due to the crossing of a spectral break or jet effects - the intensity profile is also likely to change, thus also violating assumption (iv). When the break is due to jet effects, or when the fireball becomes non-relativistic, then the index $\delta$ of the power-law form for the image radius will change. Furthermore, breaks in the light curve will produce additional uncertainties in the microlensing and afterglow parameters by introducing additional correlations. For example, in the case of a single power-law flux decline, the impact parameter $b$ can be found by extrapolating the late-time light curve (when the afterglow is not lensed) to very early times, when the afterglow is lensed by an amount that depends only on $b$. Clearly, this procedure becomes more uncertain when the light curve exhibits a break. The assumption of circular symmetry may be crude at best, especially for collimated outflows. For single lenses, it is clear that the information gathered about the surface brightness profile is inherently one-dimensional, and therefore departures from circular symmetry will be difficult to detect. Therefore, the surface brightness profile inferred assuming circular symmetry will be biased. In principle, binary lenses or single lenses with external shear would provide more information about departures from circular symmetry than a simple single lens; in practice, however, the additional freedom introduce by the more complex lens models may make extracting this information difficult. Our assumption of small optical depth is likely to hold in the outskirts of galaxies. For sight-lines passing near the center of an intervening galaxy, the magnification structure of the lens is unlikely to be well-represented by any of the three lenses we considered: an isolated single lens, a single lens with external shear, or a binary-lens. In the high-optical depth regime, it may be difficult to reconstruct the relative intensity profile directly, due to the complexity of the magnification structure. Finally, the assumption that the covariances between the intensity profile parameters and the afterglow and microlensing parameters are small is unlikely to hold in practice. This means that the errors we calculate are effectively lower limits to the true errors. The effects of these various assumptions on the resultant errors on the recovered intensity profile are interesting and important topics for future study.

Our basic results are encouraging. Errors of $1 \%$ would likely provide very stringent constraints on afterglow models, and even errors of $\sim 10 \%$ would be interesting (see Fig. 2 in Granot \& Loeb 2001). Errors of the latter magnitude can be obtained from events with larger impact parameters. For the specific afterglow parameters adopted above, we find errors of $\delta \mathcal{I} / \mathcal{I} \sim 6 \%, 10 \%, 20 \%$ in the $U$-band for impact parameters in units of $\theta_{\mathrm{E}}$ of $b=2,3$ and 4 . Since the number of expected events scales as $b^{2}$, this implies that interesting results 
might be obtained for a significant sample of afterglows. The forthcoming Swift satellite ${ }^{3}$ could provide hundreds of afterglow targets per year. A network of $1 \mathrm{~m}$ class telescopes similar to that used in gravitational microlensing searches of the Local Group (Albrow et al. 1998; Rhie et al. 1999) would provide an ideal method for "alerting" the community to microlensed afterglows, which could in turn be intensively monitored to provide information about the structure of the afterglow image. Although this represents a considerable expenditure of resources, the information gained would be invaluable, and furthermore cannot be currently acquired by any other method.

\section{Acknowledgements}

We thank Andrei Gruzinov for helpful discussions. This work was supported in part (for SG) by NASA through a Hubble Fellowship grant from the Space Telescope Science Institute, which is operated by the Association of Universities for Research in Astronomy, Inc., under NASA contract NAS5-26555, and (for AL) by grants from the Israel-US BSF (BSF-9800343), NSF (AST-9900877), and NASA (NAG5-7039).

\section{REFERENCES}

Afonso, C., et al. 2000, ApJ, 532, 340

Albrow, M., et al. 1998, ApJ, 509, 687

Albrow, M., et al. 1999a, ApJ, 522, 1011

Albrow, M., et al. 1999b, ApJ, 522, 1022

Albrow, M., et al. 2000, ApJ, 534, 894

Albrow, M., et al. 2001a, ApJ, 549, 000

Albrow, M., et al. 2001b, ApJ, 550, L173

Alcock, C., et al. 1996, ApJ, 463, L67

Alcock, C., et al. 1997, ApJ, 491, 436

\footnotetext{
${ }^{3}$ Planned for launch in 2003; see http://swift.sonoma.edu/
} 
Alcock, C. et al. 2000, ApJ, 542, 281

Blaes, O.M., \& Webster, R.L. 1992, ApJ, 391, L63

Blandford, R. D., \& McKee, C. F. 1976, Phys. Fluids, 19, 1130

Castro, S.M., Pogge, R.W., Rich, R.M., DePoy, D.L., \& Gould, A. 2001, ApJ, 548, L197

Chang, K., \& Refsdal, S. 1979, Nature, 282, 561

di Stefano, R., \& Perna, R. 1997, ApJ, 488, 55

Fruchter, A.S., et al. 1999, ApJ, 516, 683

Frail, D.A., et al. 1997, Nature, 389, 261

Freedman, D. L., \& Waxman, E. 1999, ApJ, submitted (astro-ph/9912214)

Fukugita, M., Hogan, C. J., \& Peebles, P. J. E. 1998, ApJ, 503, 518

Gaudi, B.S., \& Gould, A. 1999, ApJ, 513, 619

Gaudi, B.S., Granot, J., \& Loeb, A. 2001, ApJL, in preparation

Garnavich, P.M., Loeb, A., \& Stanek, K.Z. 2000, ApJ, 544, L11

Goodman, J. 1997, New Astronomy, 2, 449

Gould, A. 2001, PASP, in press (astro-ph/0103516)

Granot, J., \& Loeb, A. 2001, ApJ, 551, L63

Granot, J., Piran, T., \& Sari, R. 1999a, ApJ, 513, 679

1999b, ApJ, 527, 236

Halpern, J., et al. 2000, ApJ, 543, 697

Heyrovský, D. 2001, ApJ, submitted

Heyrovský, D., Sasselov, D., \& Loeb, A. 2000, ApJ, 543, 406

Katz, J., \& Piran, T. 1997, ApJ, 490, 772

Koopmans, L.V.E., \& Wambsganss, J. 2001, MNRAS, in press (astro-ph/0011029) 
Kulkarni, S.R., et al. 2000, in AIP Conf. Proc., Gamma-Ray Bursts: 5th Huntsville Symposium, ed. R. M. Kippen, R. S. Mallozzi, \& G. J. Fishman (New York: AIP)

Loeb, A., \& Perna, R. 1998, ApJ, 495, 597

Loeb, A., \& Sasselov, D. 1995, ApJ, 449, L33

Mao, S., \& di Stefano, R. 1995, ApJ, 440, 22

Mao, S., \& Loeb, A. 2001, ApJ, 547, L97

Meszaros, P., \& Rees, M. J. 1997, ApJ, 476, 232

Paczynski, B., \& Rhoads, J. E. 1993, ApJ, 418, L5

Panaitescu, A. 2001, ApJ, in press (astro-ph/0102401)

Panaitescu, A., \& Kumar, P. 2001, ApJ, in press (astro-ph/0010257)

Panaitescu, A., \& Meszaros, P. 1998, 493, L31

Pian, E., et al. 1998, ApJ, 492, L103

Press, W.H., \& Gunn, J.E. 1973, ApJ, 185, 397

Rhie, S.H., Becker, A.C., Bennett, D.P., Fragile, P.C., Johnson, B.R., King, L.J., Peterson, B.A., \& Quinn, J. 1999, ApJ, 522, 1037

Rhoads, J. E. 1997, ApJ, 487, L1

Rhoads, J., \& Fruchter, A.S. 2000, ApJ, in press

Sagar, R., Mohan, V., Pandey, S.B., Pandey, A.K., Stalin, C.S., \& Tirado, A.J. 2000, BASI, 28, 499

Sasselov, D. 1997, in Variable Stars and the Astrophysical Returns of Microlensing Surveys, ed. R. Ferlet, J.-P. Maillard, \& B. Raban (Gif-sur-Yvette: Editions Frontières), 141

Sari, R. 1998, ApJ, 494, L49

Sari, R., Piran, T., \& Narayan, R. 1998, ApJ, 497, L17

Schneider, P., \& Weiss, A. 1986, 164, 237

Schneider, P., Ehlers, J., \& Falco, E.E. 1992, Gravitational Lenses (Heidelberg: Springer), p. 313 
Udalski, A., Szymański, M., Kalużny, J., Kubiak, M., Mateo, M., Krzemiński, W., \& Paczyński, B. 1994, AcA, 44, 227

Valls-Gabaud, D. 1995, in Large Scale Structure of the Universe, ed. J.P. Mücket, S. Gottlöber, \& V. Müller (Singapore: World Scientific), 326

Wang, X., \& Loeb, A. 2000, ApJ, 535, 788

Wijers, R. A. M. J., \& Galama, T. J. 1999, ApJ, 523, 177

Witt, H. 1990, A\&A, 236, 311

Witt, H.J., \& Mao, S. 1994, ApJ, 430, 505

Wambsganss, J. 1997, MNRAS, 284, 172

Waxman, E. 1997a, ApJ, 485, L5

Waxman, E. 1997b, ApJ, 489, L33

Waxman, E. 1997c, ApJ, 491, L19

Waxman, E., Kulkarni, S. R., \& Frail, D. A. 1998, ApJ, 497, 288 


\begin{tabular}{|c|c|c|c|}
\hline Filter & $\begin{array}{c}\text { Apparent } \\
\text { Magnitude }^{1}\end{array}$ & $\begin{array}{c}\mu_{\text {sky }} \\
\left(\mathrm{mag} \mathrm{asec}^{-2}\right)\end{array}$ & $\begin{array}{c}\Gamma_{\nu} / A_{\mathrm{T}}{ }^{2} \\
\left(\gamma \mathrm{s}^{-1} \mathrm{~m}^{-2}\right)\end{array}$ \\
\hline \hline$U$ & 20.1 & 22.8 & 43 \\
$B$ & 20.6 & 22.5 & 77 \\
$V$ & 20.1 & 21.5 & 78 \\
$R$ & 19.7 & 20.8 & 130 \\
$I$ & 19.3 & 19.3 & 128 \\
$J$ & 18.3 & 15.9 & 194 \\
$K$ & 16.8 & 13.7 & 540 \\
\hline
\end{tabular}

Table 1 Adopted fluxes for GRB 000301C.

${ }^{1}$ Demagnified apparent magnitude of GRB 000301C extrapolated to 1 day after the burst.

${ }^{2}$ Number of photons per second per square meter of collecting area $A_{\mathrm{T}}$. 


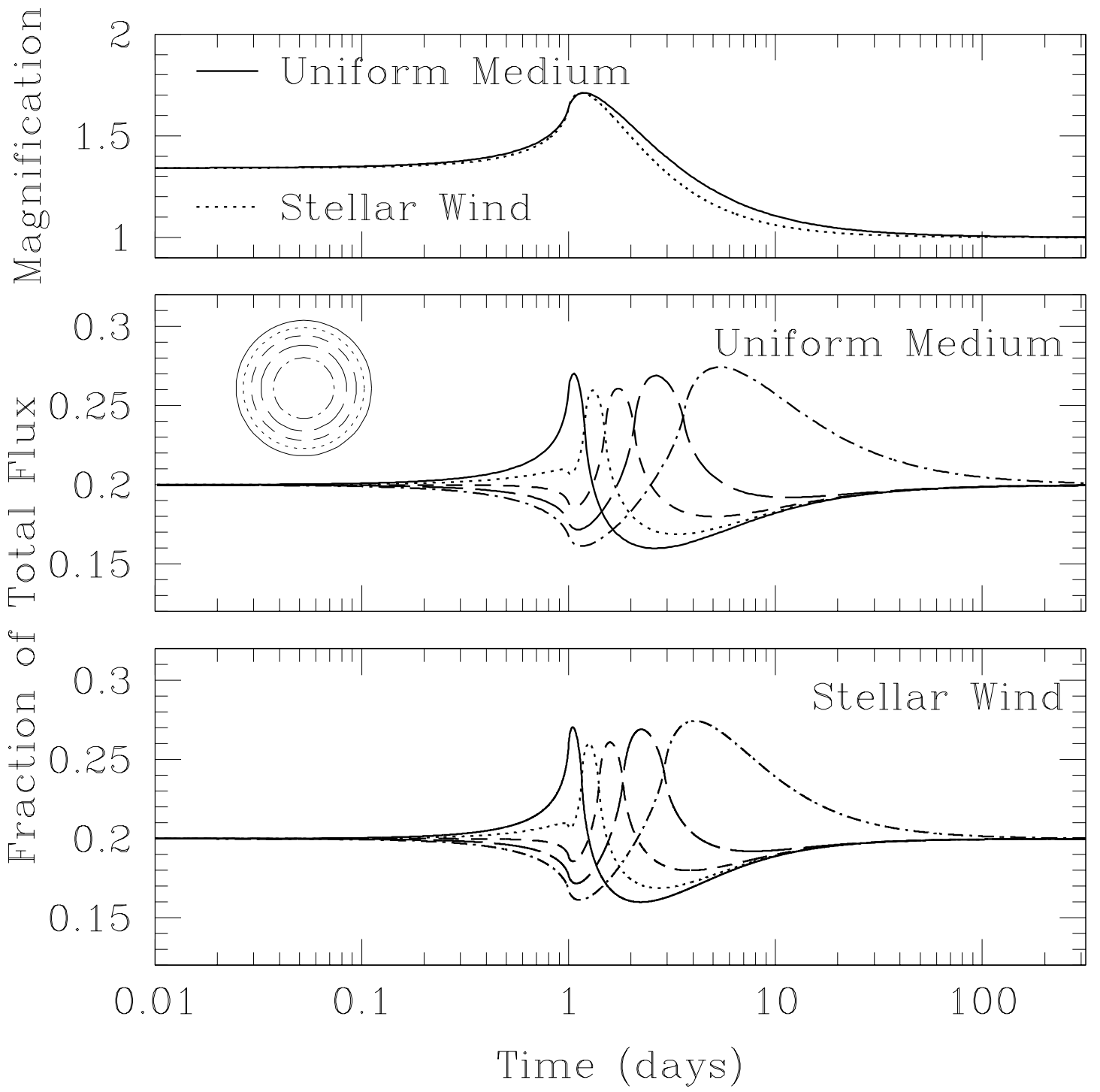

Fig. 1. - The top panel shows the magnification as a function of time for a uniform source with radius $R_{0}=1$ at $t=1$ day and an impact parameter $b=1$, assuming a uniform external medium (solid line, $\rho=$ constant) and a medium such as that created by a stellar wind (dotted line, $\rho \propto r^{-2}$ ). The middle panel shows the fraction of total flux contributed by five equal area annuli as a function of time in days for the uniform external medium, while the lower panel shows the same but for the stellar wind medium. 

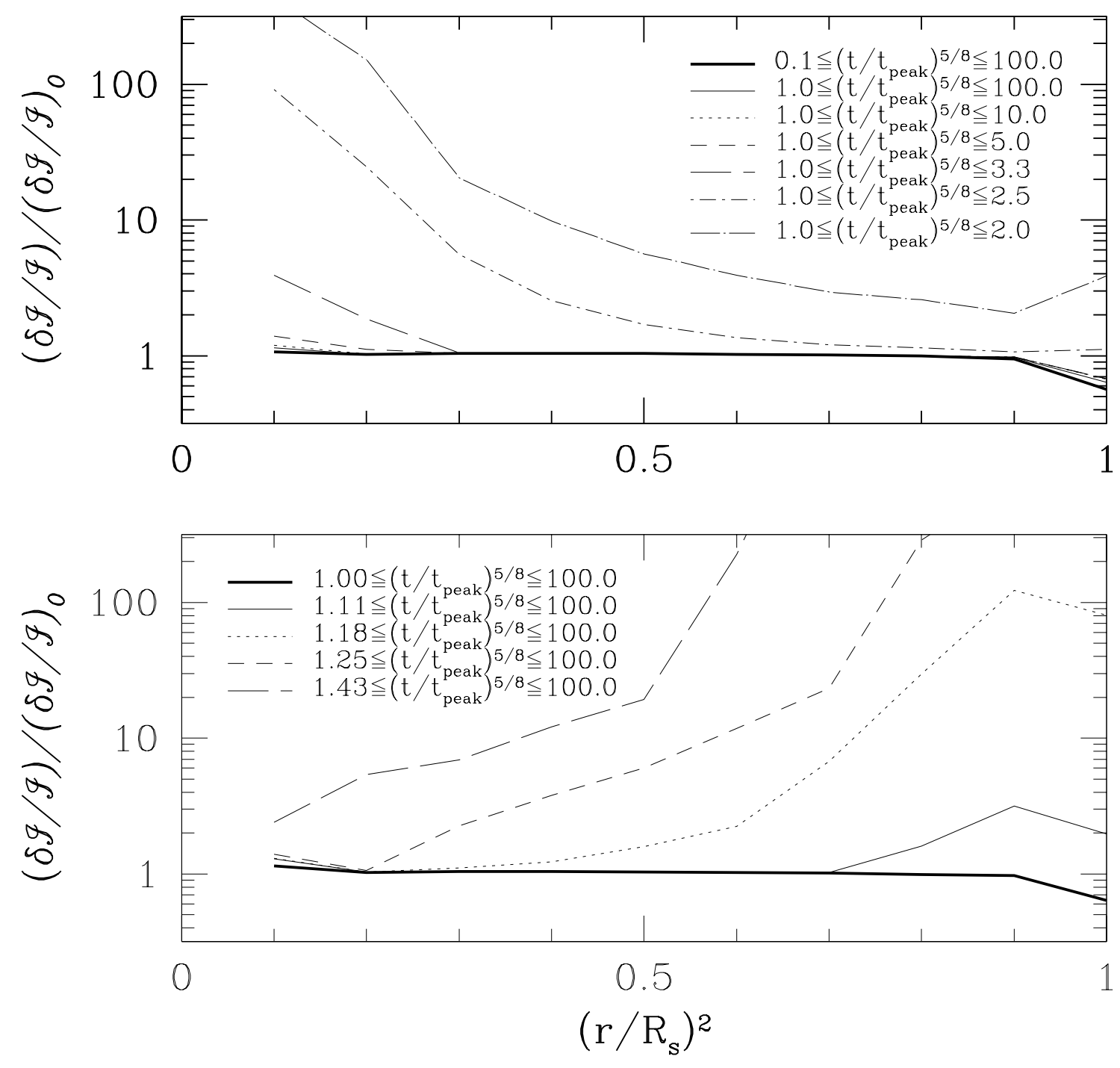

Fig. 2.- Both panels show the fractional error in the recovered intensity profile $\delta \mathcal{I} / \mathcal{I}$ normalized to the fiducial error $(\delta \mathcal{I} / \mathcal{I})_{0}$ given by the scaling relation in equation $(13)$, as a function of the square of the normalized radius $X=r / R_{\mathrm{s}}$ of the afterglow. Each line shows various assumptions of the beginning and duration of observations relative to the time $t_{\text {peak }}$ of the peak of the microlensing event. Top Panel: Varying the end of observations relative to $t_{\text {peak. }}$ Bottom Panel: Varying the beginning the observations relative to $t_{\text {peak }}$. 

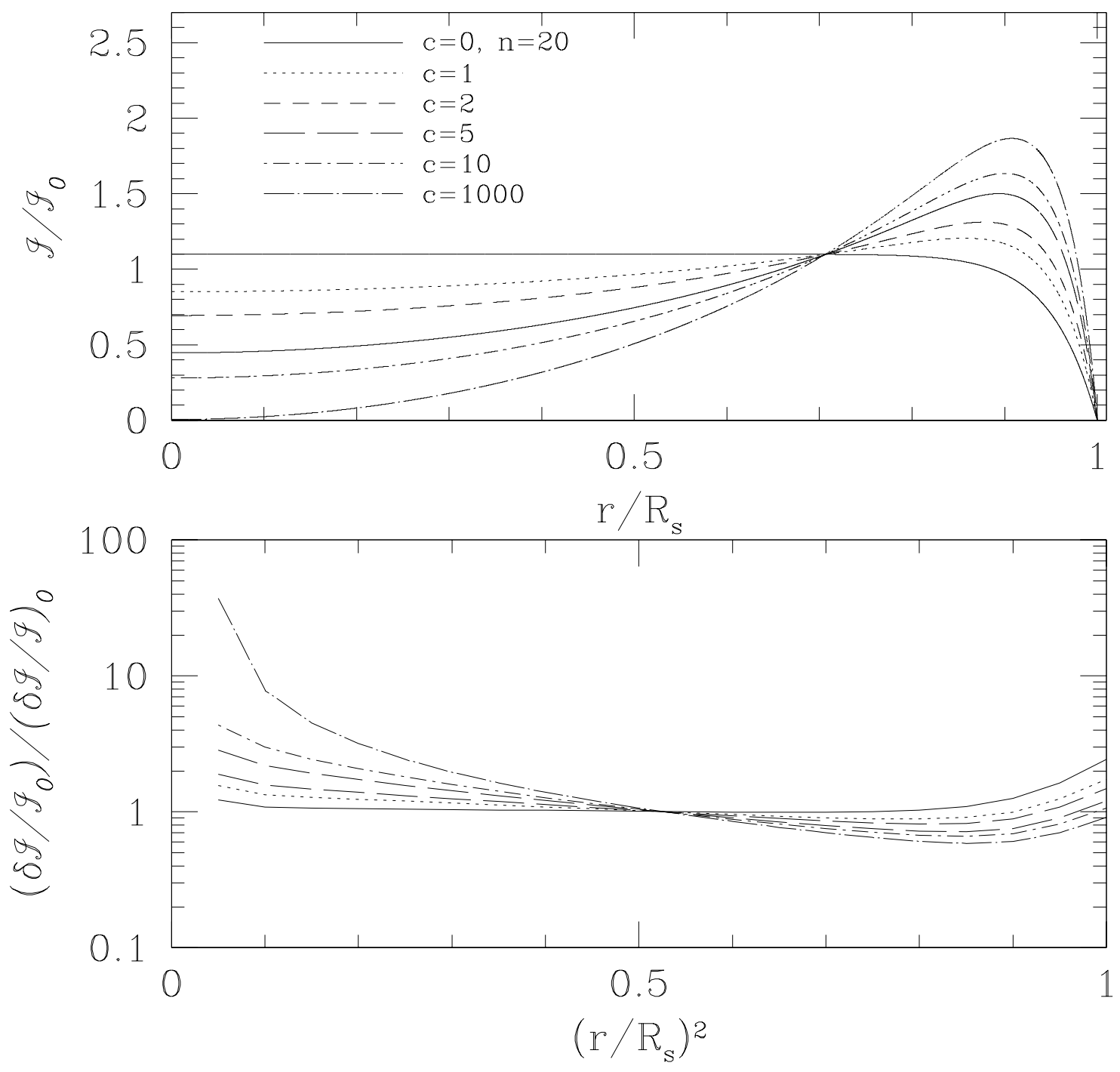

Fig. 3.- Top Panel: The normalized intensity profile $\mathcal{I}(r) / \mathcal{I}_{0}$ of the source as a function of the normalized radius $X=r / R_{\mathrm{s}}$, assuming a cutoff parameter $n=20$ and for several different values of the parameter $c$ (see text and equation (14) for details). Bottom Panel: The fractional error in the recovered intensity profile $\delta \mathcal{I} / \mathcal{I}_{0}$ normalized to the fiducial error $(\delta \mathcal{I} / \mathcal{I})_{0}$ given by the scaling relation in equation (13), as a function of the square of the normalized radius of the afterglow, for the intensity profiles shown in the top panel. 


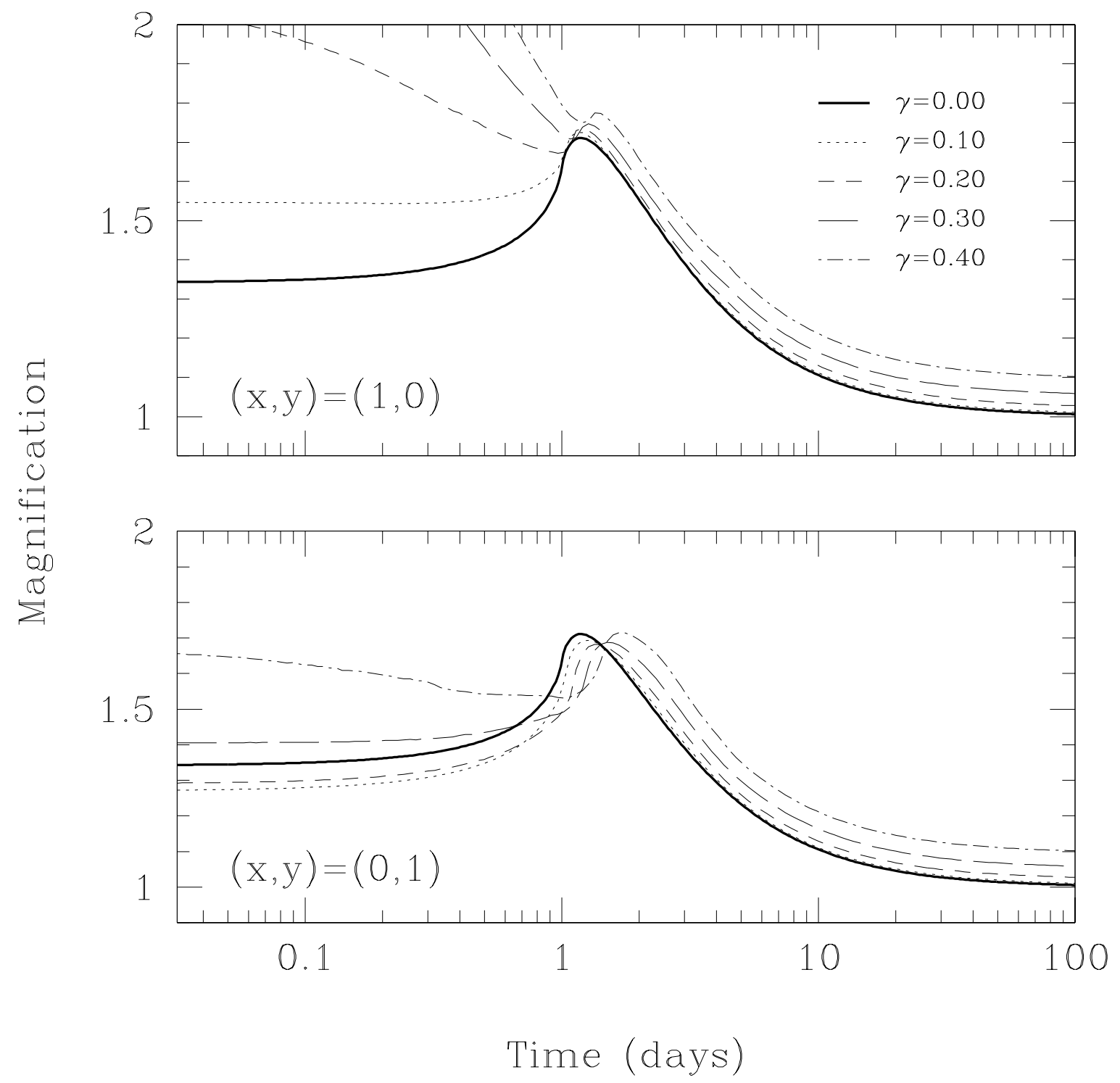

Fig. 4. - Magnification as a function of time for a uniform source with $R_{0}=1$ and $b=1$ for a single lens with external shear along with $x$-axis of magnitude $\gamma$ for various values of $\gamma$. Top Panel: Assuming the center of the source is located on the x-axis of the lens. Bottom Panel: Assuming the center of the source is located on the y-axis of the lens. 

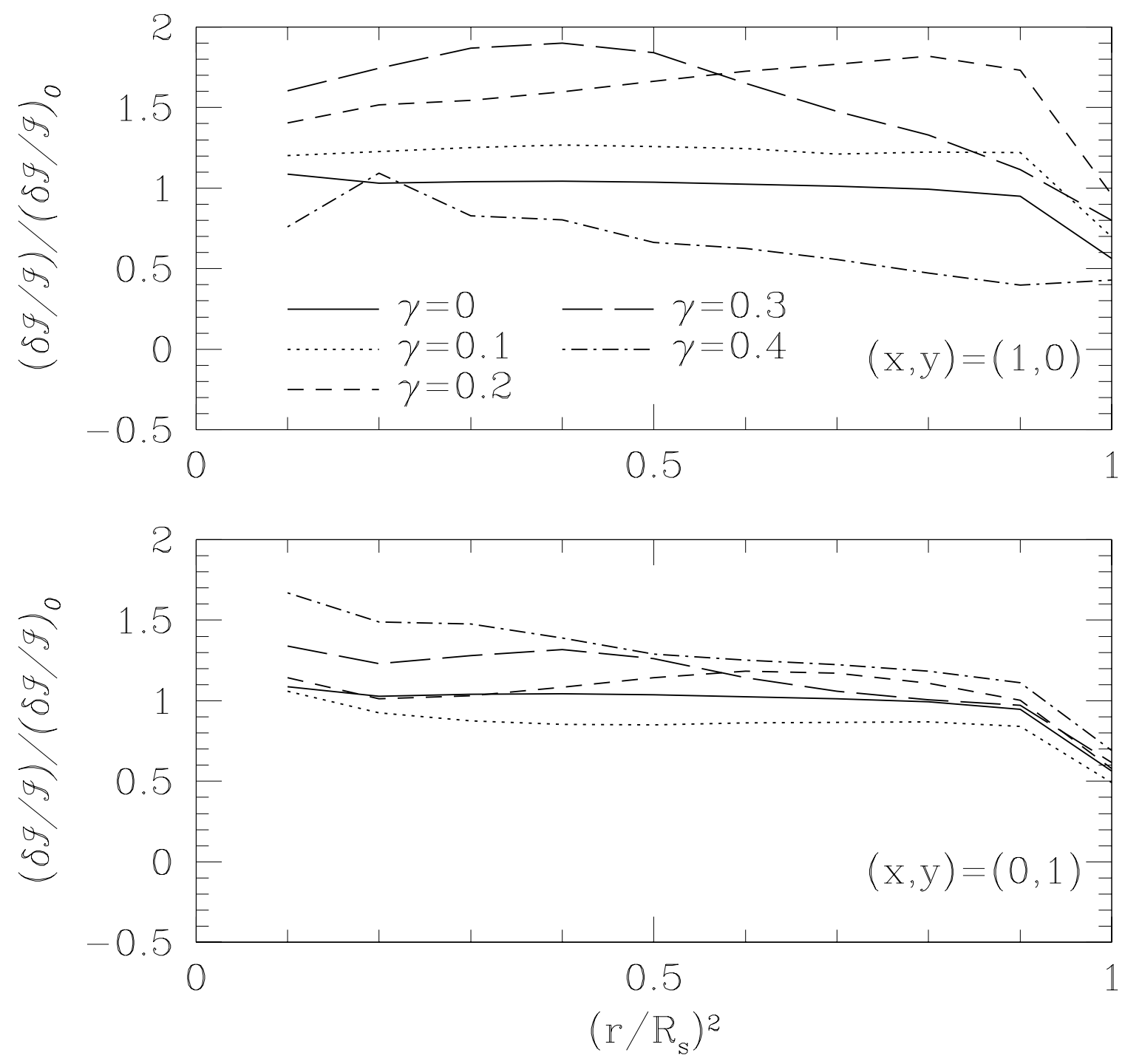

Fig. 5.- The fractional error in the recovered intensity profile $\delta \mathcal{I} / \mathcal{I}$ normalized to the fiducial error $(\delta \mathcal{I} / \mathcal{I})_{0}$ given by the scaling relation in equation (13), as a function of the square of the normalized radius $X=r / R_{\mathrm{s}}$ of the afterglow for various values of the external shear $\gamma$ along the x-axis. We have assumed $R_{0}=1, b=1$, and a uniform source. Top Panel: Assuming the center of the source is located on the x-axis of the lens. Bottom Panel: Assuming the center of the source is located on the y-axis of the lens. 

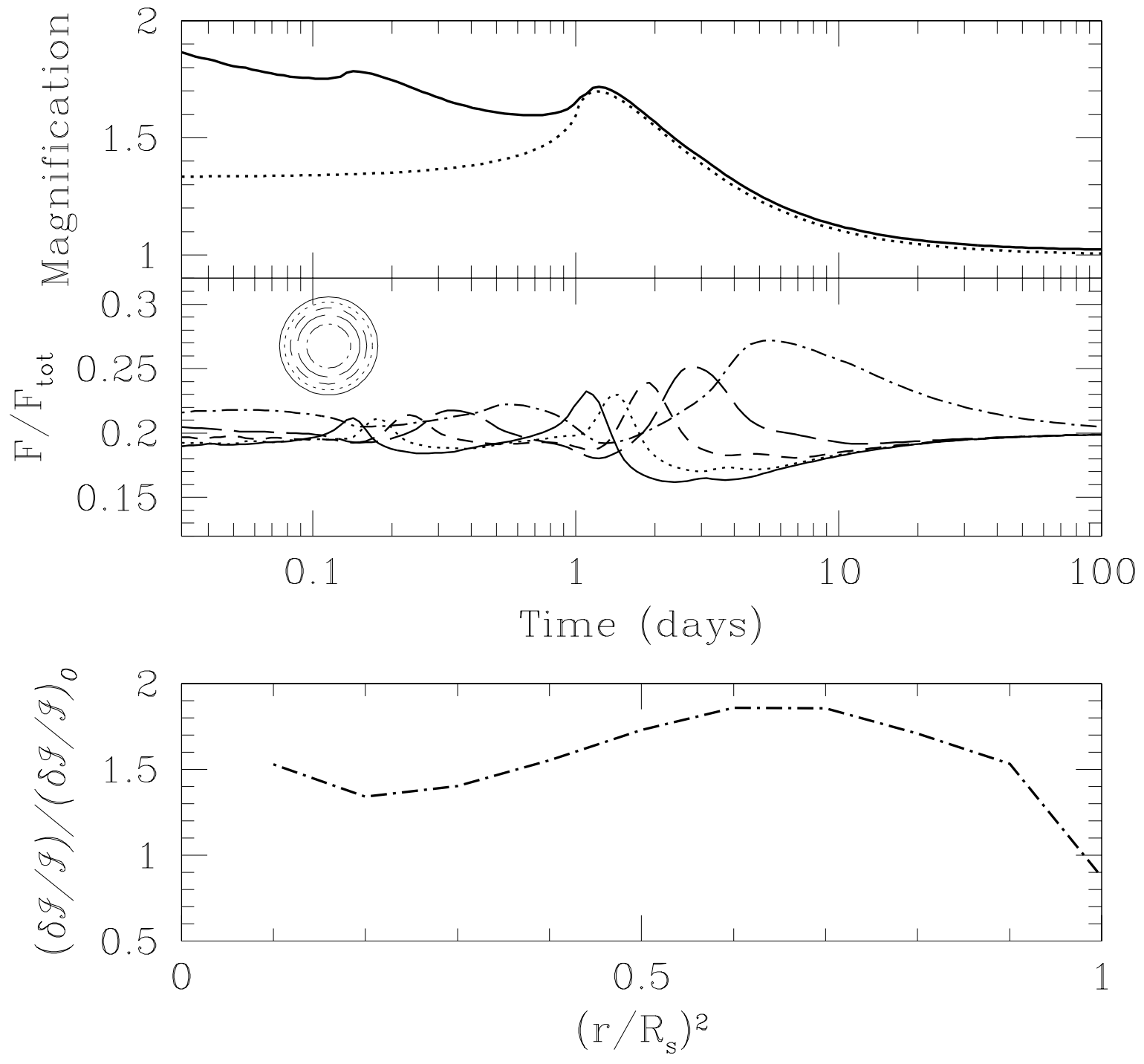

Fig. 6. - Top Panel: The magnification as a function of time for a uniform source with $R_{0}=1$. The solid line is for a binary-lens with mass ratio $q=1$ and a separation of $d=0.8$ in units of the combined Einstein ring radius. The source is centered on $(-0.16,-1)$. The dotted line is for a single lens with impact parameter $b=\left(0.16^{2}+1^{2}\right)^{1 / 2}=1.013$. Middle Panel: The fraction of total flux contributed by five equal area annuli as a function of time in days for the binary-lens light curve shown in the top panel. Bottom Panel: The fractional error in the recovered intensity profile $\delta \mathcal{I} / \mathcal{I}$ normalized to the fiducial error $(\delta \mathcal{I} / \mathcal{I})_{0}$ given by the scaling relation in equation (13), as a function of the square of the normalized radius $X=r / R_{\mathrm{S}}$ of the afterglow. 


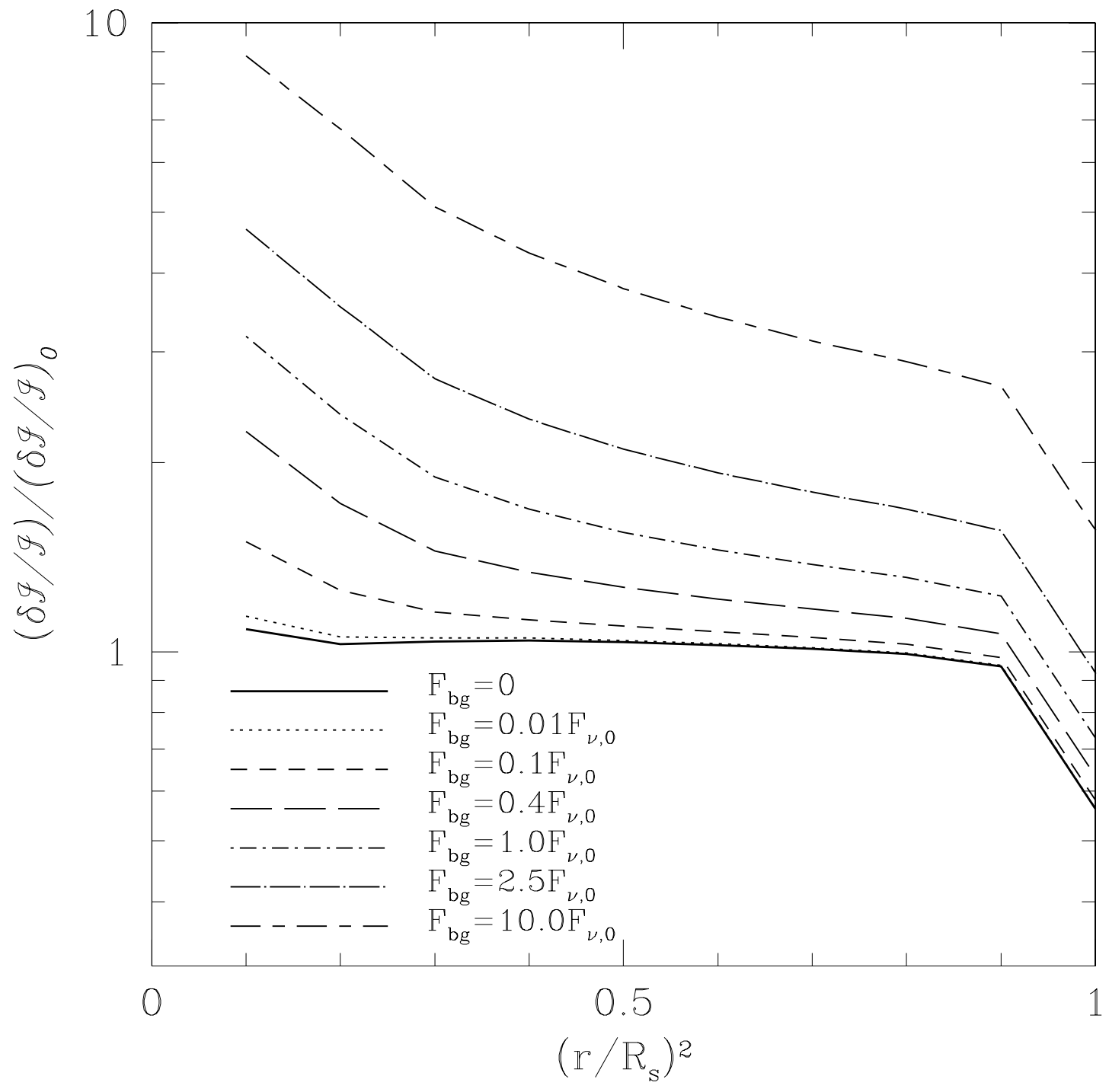

Fig. 7.- The fractional error in the recovered intensity profile $\delta \mathcal{I} / \mathcal{I}$ normalized to the fiducial error $(\delta \mathcal{I} / \mathcal{I})_{0}$ given by the scaling relation in equation (13), as a function of the square of the normalized radius of the afterglow for various assumptions about the magnitude the unresolved background flux $F_{\mathrm{bg}}$ relative to the flux $F_{\nu, 0}$ of the afterglow at $t=1$ day. 


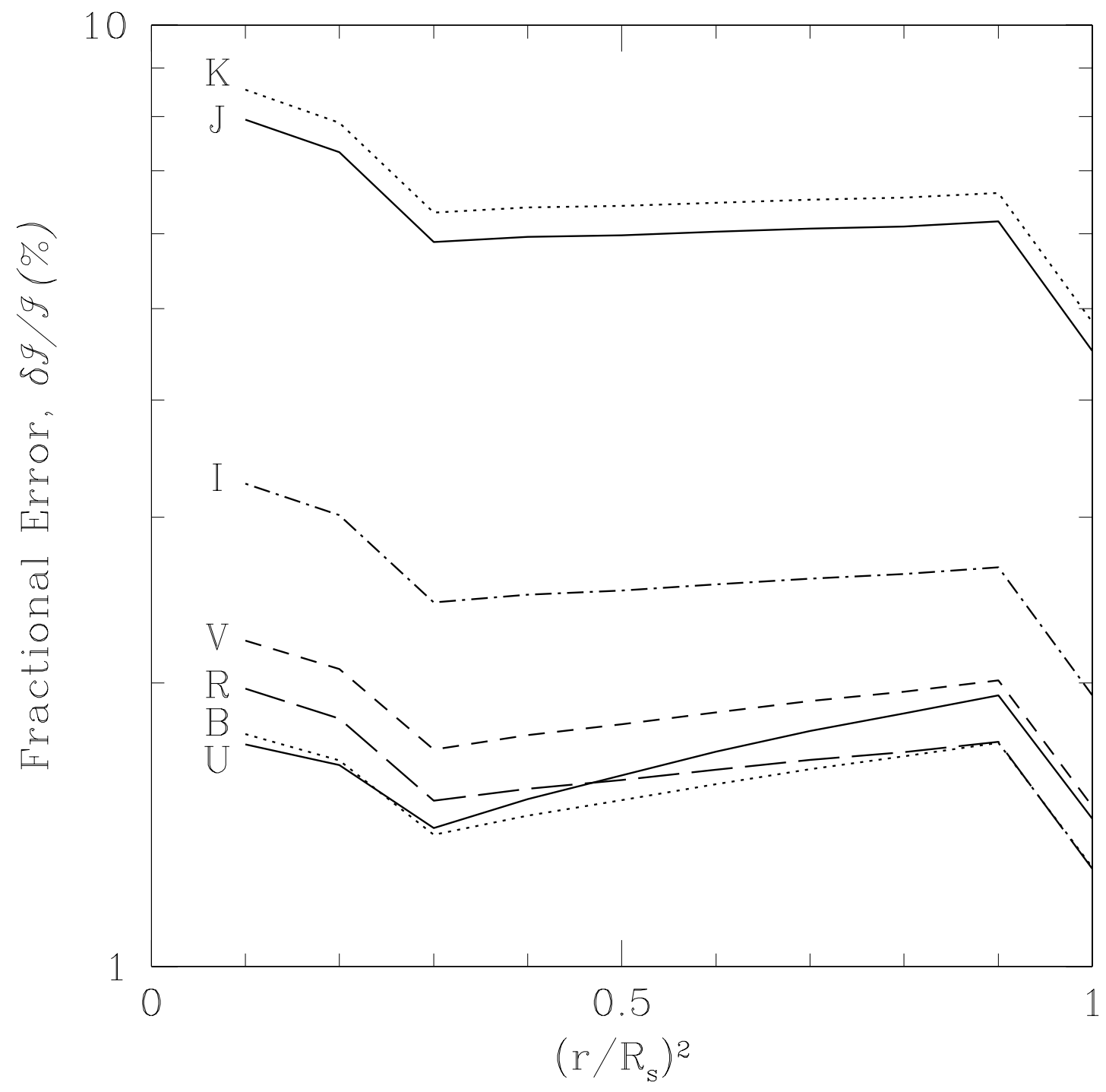

Fig. 8. - The fractional error $\delta \mathcal{I} / \mathcal{I}$ in the recovered relative intensity profile in percent as a function of the square of the normalized radius $X=r / R_{\mathrm{s}}$, for the afterglow and microlensing parameters of GRB 000301C, assuming a uniform source, and that observations were made with a $4 \mathrm{~m}$ telescope from the peak of the microlensing event until $\sim 30$ days after the peak. 


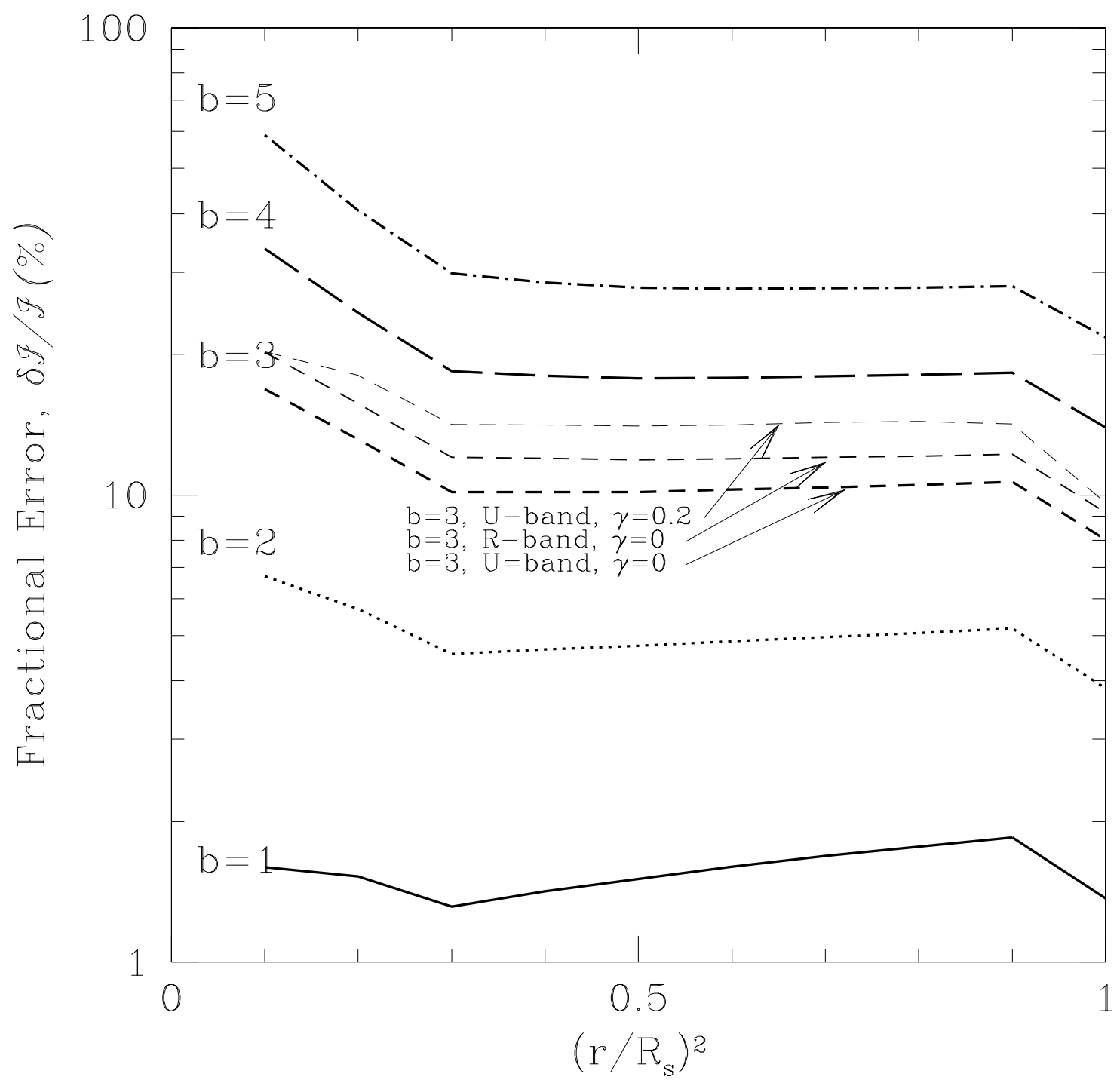

Fig. 9.- Same as Figure 8 for $U$-band, except we have varied the impact parameter of the microlensing event, $b$. Note that the fraction of all afterglows with impact parameter $\leq b$ is $\sim 1 \% b^{2}$. 